Hepatic Encephalopathy. By ALAN H LOCKWOOD. (Pp 151; Price: £52.00). 1992. Oxford, Butterworth-Heinemann. ISBN 0-7506-9234-0.

This monograph appears to be intended for the specialist with an interest in liver disease. This may explain why only 19 of the 143 pages are devoted to the clinical and laboratory features of hepatic encephalopathy, so the practising neurologist may be disappointed. A discussion of the theories about the exact nature of the metabolic abnormality that produces hepatic encephalopathy forms the bulk of this work. Such mechanisms as the role of ammonia, neurotransmitters, toxins, varied amino-acid concentrations and the GABA-benzodiazepine receptor complex are explored in detail. The author has quantitatively studied ammonia metabolism by following the clearance of isotopically labelled ammonia in controls and patients with liver disease. $\mathrm{He}$ has stressed the importance of skeletal muscle as a major organ in maintaining normal blood ammonia levels. Clearly, arterial ammonia levels are raised in hepatic coma. $\mathrm{He}$ discusses the evidence to support the role of ammonia in hepatic encephalopathy emphasising the established clinical and experimental evidence.

There is an excellent section (18 pages) on treatment: again discussing the well tested methods-protein restriction and diet, the addition of lactulose (and lactitol), neomycin and its combination with lactulose. Less well tested therapies such as branched chain amino-acids and the use of neurotransmitters e.g. L-dopa and bromocriptine, are considered, although the author is not convinced of their efficacy. Finally he describes the evidence for the use of the benzodiazepine antagonist flumazenil.

This is a useful small book in a restricted field and will find a deserved place on the shelves in the liver unit.

T FOWLER

Benign Localized and Generalized Epilepsies of Early Childhood (Epilepsy Research Supplements/Vol. 6) By R DEGEN AND FE DREIFUSS. (Pp 230; Price: US\$205.50/Dfl.360.00). 1992. Amsterdam, Elsevier Science Publishers B.V. New York, Elsevier Science Publishers B.V. ISBN 0-444-81266-0.

The purpose of this book is to better define the rather confused and difficult area of localized and generalized epilepsies in early and middle childhood. The contents are divided into 32 chapters with 72 authors contributing, some of whom are the most distinguished in the field of epilepsy. Unfortunately, because of the large number of contributing authors, the variation in information and quality between chapters is significant. Some chapters are excellent, giving detailed reviews of previous literature, and summaries of the current state of knowledge. Others are simply short papers covering only very specific study which that particular author(s) happened to research.

Most confusing was the use of different terminology, especially concerning benign childhood epilepsy with centrotemporal spikes. Each chapter which addresses this subject uses different terminology and abbreviations. The last chapter or summation was essential to bring order to this smorgasbord of information. The editors, however, apologize in the introduction for the different styles and quality of the chapters, but say that they do not wish to violate the integrity of the authors. On the other hand, it is the reader who spends the money to buy the book and who expects to understand the childhood epilepsies after reading it. Therefore, I feel more rigorous editing would have been appropriate, at least to coordinate terminology and content.

This book is not for the medical student or beginner in epilepsy. For a paediatric neurologist or epileptologist, however, it can be a worthwhile acquisition especially for a reference library.

\section{ELINOR BEN-MENACHEM}

A Textbook of Epilepsy. 4th Edition. Edited by J LAIDLAW, A RICHENS AND D CHADWICK. (Pp 754 Illustrated; Price: £100.00). 1992. Edinburgh, Churchill Livingstone. ISBN 0-443-04473-2.

By assembling a very strong cross-Atlantic team, the editors of this distinguished book-4 editions in 16 years - have produced a considerably expanded and very up-to-date work of reference which teems with clinical experience and expertise. The interests of the patient remain paramount throughout all the advice given regarding investigation and management of seizures. There is little repetition, it is very readable, controversial classifications though dealt with, are minimised, and some contributions are monographs in themselves. These include the section on epilepsy in childhood, with 370 references, that on E.E.G. (400) and the psychiatric chapter. The importance of age, even in days, in infantile seizure syndromes, and the frequency in the over-75s may surprise some.

The combination of the E.E.G., videocamera and telemetric recording has transformed the investigation of certain seizures, part only of an outstanding account of the valuable uses (and useless abuses) of electroencephalography. The lines of thought governing the psychiatric chapter should be carefully read, and applied to many medical conditions, while the magnificently illustrated radiology section abounds with modern technologies yet still appreciates that not everyone has access to them, so that isotope encephalography and even air-encephalography are not cast scournfully aside.

The use of brain slices as a research tool in studying origins and spread of epileptic discharges, and the effects of drugs, may be unfamiliar to some clinicians, but the recommendations to them against polytherapy come through loud and clear. The legal chapters make absorbing reading but as always leave many unsolved problems. It is sad that for so excellent a work of reference the index ( 4 out of 478 pages) is so woefully inadequate, and proofing errors abound, even astonishingly extending to the table of contents and some figures. The type used for headings and sub-headings is irritatingly inconsistent, as is the use of the terms fig. or tab. for tabs. However it is a book essential to neuroscience departments, and fund holders should note its great value to those who deal frequently, but not day-by-day with epilepsy. The charming ave-atque-vale epilogue by the Laidlaws is the icing on a rich and highly digestible cake.

ER BICKERSTAFF
Surgical Neuro-Angiography/4 Endovascular Treatment of Cerebral Lesions. By A BERENSTEIN and P LASJAUNIAS. $\quad(\mathrm{Pp} \quad 344$ Illustrated; Price: DM298.00). Heidelberg, Springer-Verlag. 1992. ISBN 3-540-17500-8.

Surgical (or Interventional) Neuroradiology is one of the most rapidly developing areas of modern medicine. Vascular techniques (Surgical Neuro-angiography) form its largest part. After almost three decades as a distinct clinical discipline the time has come for a critical review of the rationale (or philosophies), techniques and consequences of these treatments.

Bravely, Alex Berenstein and Pierre Lasjaunias have undertaken this overview in their planned series of five volumes. Previous volumes cover the functional anatomy of the cranio-facial arteries, endovascular treatment of cranio-facial lesions and the functional vascular anatomy of the brain, spinal cord and spine. After the present volume on the Endo-vascular Treatment of Cerebral Lesions, a fifth volume is planned on the Endo-vascular Treatment of the Spine and Spinal cord lesions, with added sections on the management of subarachnoid haemorrhage including intracranial angioplasty for vasospasm and the embolisation of aneurysms.

Berenstein and Lasjaunias are eminently qualified to undertake this overview. Their working lives cover completely the evolution of modern techniques and their case experience is one of the largest world-wide. Their stated aim for their fourth volume is geared to "understanding the vascular abnormalities of the brain and pin-pointing when and how vascular interventional Neuro-angiography of cerebro-vascular problems should be undertaken". They achieve their aim in a presentation of typically high quality, copiously illustrated ( 375 illustrations in 203 Figures) and referenced in detail (more than five hundred references up to 1992). The value of this work is enhanced by the authors' critical attitudes and by insights and perceptions in their work that can come only from an intimate knowledge of their subject.

This volume will be read avidly by all interventional neuroradiologists. Since intracranial vascular malformations can be managed well only through close co-operation between neurosurgeons, surgical neuroradiologists and radiotherapists, the book should also be required reading for every Neurosurgeon and Radiotherapist in this field. The complete series of Surgical Neuro-angiography should have a place in every library for the Neuro-sciences, Neurosurgery and Surgical Neuroradiology.

M NELSON

Surgical Treatment of Epilepsy. (Epilepsy Research Supplement 5, 1992). Edited by WM H THEODORE. (Pp 250; Price: US\$200.00/Dfl.320.00). Amsterdam, Elsevier Science Publishers. 1992. ISBN 0444-89474-8.

This monograph, comprising 30 chapters in 250 pages is well organised and well produced. It was born of an idea in the United States that with the growth of the surgical treatment of epilepsy there was a need for some kind of minimal consensus about the preoperative selection, surgical procedures 